\title{
Assessing the safety and immunogenicity of recombinant vesicular stomatitis virus Ebola vaccine in healthy adults: a randomized clinical trial
}

\author{
May S. ElSherif MD MPH, Catherine Brown BN RN, Donna MacKinnon-Cameron MMath, Li Li MSc, Trina Racine \\ PhD, Judie Alimonti PhD, Thomas L. Rudge PhD, Carol Sabourin PhD, Peter Silvera PhD, Jay W. Hooper PhD, \\ Steven A. Kwilas PhD, Nicole Kilgore MS, Christopher Badorrek PhD, W. Jay Ramsey MD PhD, D. Gray Heppner MD, \\ Tracy Kemp MPH, Thomas P. Monath MD, Teresa Nowak BS, Shelly A. McNeil MD, Joanne M. Langley MD MSc, \\ Scott A. Halperin MD; on behalf of the Canadian Immunization Research Network
}

Cite as: CMAJ 2017 June 19;189:E819-27. doi: 10.1503/cmaj.170074

CMAJ Podcasts: author interview at https://soundcloud.com/cmajpodcasts/170074-res

\begin{abstract}
BACKGROUND: The 2013-2016 Ebola virus outbreak in West Africa was the most widespread in history. In response, alive attenuated recombinant vesicular stomatitis virus (rVSV) vaccine expressing Zaire Ebolavirus glycoprotein (rVSVAGZEBOV-GP) was evaluated in humans.
\end{abstract}

METHODS: In a phase 1 , randomized, dose-ranging, observer-blind, placebocontrolled trial, healthy adults aged 18-65 years were randomized into 4 groups of 10 to receive one of 3 vaccine doses or placebo. Follow-up visits spanned 180 days postvaccination for safety monitoring, immunogenicity testing and any rVSV virus shedding.
RESULTS: Forty participants were injected with rVSV $\triangle$ G-ZEBOV-GP vaccine $(n=30)$ or saline placebo $(n=10)$. No serious adverse events related to the vaccine or participant withdrawals were reported. Solicited adverse events during the 14-day follow-up period were mild to moderate and self-limited, with the exception of injection-site pain and headache. Viremia following vaccination was transient and no longer detectable after study day 3 , with no virus shedding in saliva or urine. All vaccinated participants developed serum immunoglobulin $\mathrm{G}$ (IgG), as measured by Ebola virus envelope glycoprotein-based enzymelinked immunosorbent assay (ELISA).
Immunogenicity was comparable across all dose groups, and sustained IgG titers were detectable through to the last visit, at study day 180 .

INTERPRETATION: In this phase 1 study, there were no safety concerns after a single dose of rVSVDG-ZEBOV-GP vaccine. IgG ELISA showed persistent high titers at 180 days postimmunization. There was a period of reactogenicity, but in general, the vaccine was well tolerated. This study provides evidence of the safety and immunogenicity of rVSV $\triangle$ G-ZEBOV-GP vaccine and importance of its further investigation. Trial registration: ClinicalTrials.gov no., NCT02374385
$\mathrm{T}$ he 2013-2016 Ebola virus disease (EVD) outbreak in West Africa was the most widespread in history. ${ }^{1}$ The outbreak started in December 2013 in Guinea and rapidly expanded to other countries in the region, with $>11000$ deaths in nearly 30000 cases. ${ }^{2}$ At the onset of the outbreak, several Ebola vaccines were under development; however, few had progressed to clinical trials.

An EVD vaccine, rVSV $\Delta$ G-ZEBOV-GP, was developed at the Canadian National Microbiology Laboratory of the Public Health
Agency of Canada using the live, attenuated recombinant vesicular stomatitis virus (rVSV) backbone. ${ }^{3}$ Consequent to substituting its glycoprotein (GP) gene with that of a target pathogen, live, attenuated rVSV synthesizes and expresses foreign viral GP antigens, which subsequently induce cellular and humoral immunity. ${ }^{4}$ Wild-type VSV infects primarily cattle and horses, but rarely causes clinical infections in humans. ${ }^{5,6}$ In vivo, the rVSV is highly attenuated and nonpathogenic, with narrower cell tropism than wild-type VSV; ${ }^{7,8}$ it replicates normally, expressing the 
imported GP that binds to host cell receptors and initiates immune responses. ${ }^{9}$

rVSV $\triangle$ G-ZEBOV-GP provides both pre- and postchallenge protection in animal models. ${ }^{10,11}$ In nonhuman primates, the vaccine was well tolerated ${ }^{12}$ and protective against lethal Zaire Ebola virus (ZEBOV) challenges following a single dose. ${ }^{13}$ The vaccine induced protective humoral and cellular immune responses in all vaccinated monkeys. ${ }^{14}$

As part of a coordinated, international effort to expeditiously evaluate candidate EVD vaccines and make them available to control the epidemic, we conducted a phase 1 trial of the rVSV $\Delta \mathrm{G}$ -
ZEBOV-GP. The main objective of this coordinated partnership was to determine the lowest vaccine virus dose that would be safe and well tolerated, and induce an immune response.

\section{Methods}

\section{Study design and participants}

This was a single-centre, randomized, observer-blind, doseranging, placebo-controlled trial to assess the safety (including rVSV viremia and shedding) and immune response after a single injection of one of 3 dose levels of the rVSV $\Delta$ G-ZEBOV-GP vaccine. ${ }^{15}$

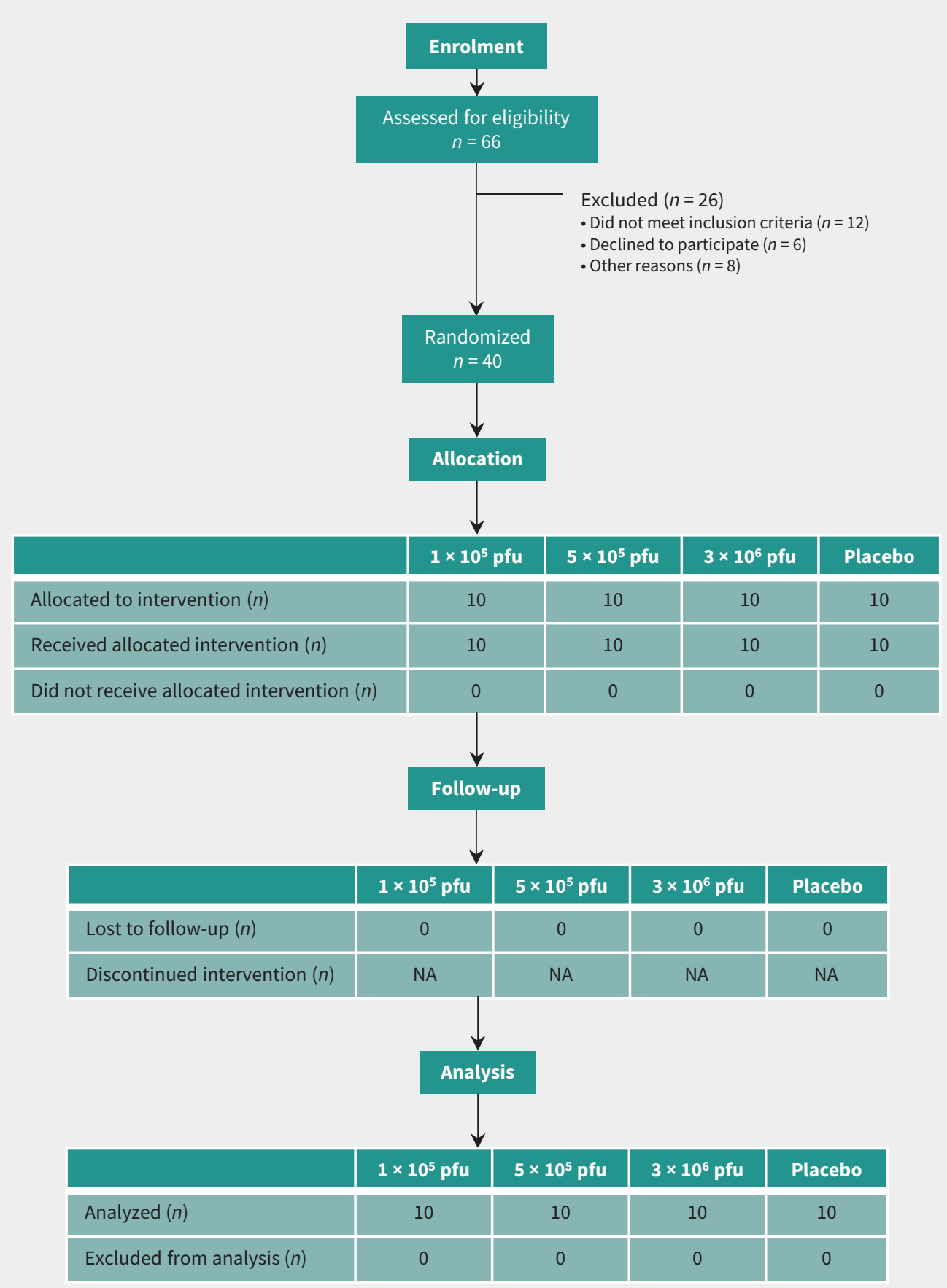

Figure 1: Trial diagram showing subjects randomized to vaccine cohorts or placebo. Note: NA= not applicable, pfu = plaque-forming units. 
Healthy adult male and female volunteers aged 18 to 65 years were randomly assigned in a 1:1:1:1 ratio to one of 3 vaccine groups $(1 \times$ $10^{5}$ plaque-forming units [pfu], $5 \times 10^{5} \mathrm{pfu}$, and $3 \times 10^{6} \mathrm{pfu}$ ) or a placebo control group (Figure 1). A randomization list was computer generated with a block size of 8 . Exclusions to enrolment included prior infection with a filovirus or VSV or risk of exposure to either; health care worker; child care worker or household contact with young children; pregnant or lactating women; immunocompromised individuals; allergy or any adverse reactions to the vaccine components or other vaccines; underlying medical conditions; and abnormalities on screening tests. Participant screening included medical history, physical examination, electrocardiogram, and blood testing, including complete blood count with white blood cell differential, prothrombin and partial thromboplastin times, serum metabolic panel, urine pregnancy screen (females only), viral serologies (hepatitis $\mathrm{B}, \mathrm{C}, \mathrm{HIV}$ ) and urinalysis. The study was conducted at the Canadian Center for Vaccinology in Halifax (clinicaltrials.gov, no. NCT02374385).

\section{Vaccine}

The rVSV $\triangle$ G-ZEBOV-GP vaccine is a live, attenuated recombinant virus in which the GP gene of VSV Indiana strain is replaced by that of the ZEBOV Kikwit 1995 strain. The vaccine was licensed to BioProtection Systems (NewLink Genetics Corporation) and more recently sublicensed to Merck \& Co., Inc. Vaccine product was compliant with good manufacturing practices, suspended in recombinant human serum albumin $2.5 \mathrm{~g} / \mathrm{L}$ and $10 \mathrm{mM}$ Tris (pH 7.2), dispensed at $1 \times 10^{8} \mathrm{pfu} / \mathrm{mL}$ per unit vial and stored at $-70^{\circ} \mathrm{C}$ (lot number 0030513). Preservativefree normal saline was used as diluent (Alveda $\mathrm{NaCl} 0.9 \%$ lot number: 13331012) to prepare lower doses. Placebo injections were $1 \mathrm{~mL}$ normal saline. Study pharmacists prepared allocated treatment; an unblinded nurse concealed and administered it. The pharmacists did not have any interaction with study participants or blinded study staff, and the unblinded nurse had no other role in the study.

\section{Study procedures}

Each participant was injected intramuscularly with $1 \mathrm{~mL}$ of vaccine or placebo. Participants were monitored at least 30 minutes postinjection for adverse events (AEs). Assessment visits occurred on days $1,3,7,14,28,56,84$ and 180 . During the 14-day period after injection, solicited and unsolicited AEs were collected using memory aids; subjects recorded temperature, injection-site reactions, serious adverse events (SAEs) or systemic reactogenicity symptoms. Solicited symptoms included injection-site redness, swelling or pain; subjective and objective fever; chills; sweats; myalgia; arthralgia; fatigue; headache; and gastrointestinal symptoms. Thereafter, unsolicited AEs were documented on days $28,56,84$ and 180 . SAEs were monitored throughout the study. The investigator $(\mathrm{SAH})$ assessed all AEs for causality.

†Race was self-reported.
The first volunteer was injected only after day 7 data from the first and lowest dose $\left(3 \times 10^{6} \mathrm{pfu}\right)$ cohort of a separate doseescalating Walter Reed Army Institute of Research (WRAIR) study ${ }^{16}$ were evaluated by the independent Data Safety Monitoring Board. This assured us that we could proceed with our dose-ranging design, as $3 \times 10^{6} \mathrm{pfu}$ was the highest dose used in this study. Other safety measures included staggered vaccination, follow-up, holding rules and safety monitoring for AEs and SAEs. The study was monitored by an Independent Research Monitor.

\section{Outcomes}

The primary outcome was vaccine safety and tolerability through assessment of injection-site events, AEs, SAEs at all visits, and hematologic and biochemical laboratory measures at days $0,1,3$, 7, 28 and 180, as well as testing for viremia and viral shedding on study days $0-14$. Secondary outcomes were antibody measurements on days $0,7,14,28,56,84$ and 180 by enzyme-linked immunosorbent assay (ELISA), and days 0 and 28 by pseudovirion neutralization assay (PsVNA) 50 and PsVNA80.

An ELISA using recombinant glycoprotein ( $\mathrm{rGP}$ ) from homologous Zaire-Kikwit strain as the solid phase assessed total anti-GP immunoglobulin G (IgG) in sera (Battelle Biomedical Research Center, West Jefferson, $\mathrm{OH}$ ), Battelle Standard Operating Procedure, BBRC. X-127. ${ }^{17-19}$ A PsVNA measured neutralizing antibody responses to Ebola GP (US Army Medical Research Institute of Infectious Diseases, Fort Detrick, MD)..$^{16,20-24}$ This assay is based on non-replicating VSV expressing luciferase reporter protein particles pseudotyped with ZEBOV envelope glycoproteins. ${ }^{20}$ The assay determined the highest serum dilutions causing $50 \%$ (PsVNA50) and 80\% (PsVNA80) inhibition of virus cell entry and expression of luciferase.
Table 1: Baseline characteristics by intervention groups*

$\begin{array}{cccccc} & \text { Vaccine, } & \text { Vaccine, } & \text { Vaccine, } & \text { All vaccine } & \\ & 1 \times 10^{5} \mathrm{pfu} & 5 \times 10^{5} \mathrm{pfu} & 3 \times 10^{6} \mathrm{pfu} & \text { subjects } & \text { Placeb } \\ \text { Characteristic } & (n=10) & (n=10) & (n=10) & (n=30) & (n=10)\end{array}$

Age

$\begin{array}{lccccc}\text { Mean } & 34.5 \pm 15.4 & 35.8 \pm 12.1 & 36.5 \pm 12.8 & 35.6 \pm 13.1 & 32.0 \pm 9.4 \\ \text { Range } & 18-61 & 22-62 & 21-56 & 18-62 & 20-50\end{array}$

Sex, $n(\%)$

$\begin{array}{lllll}\text { Male } & 3(30) & 5(50) & 4(40) & 12(40)\end{array}$

$\begin{array}{lllll}\text { Female } & 7(70) & 5(50) & 6(60) & 18(60)\end{array}$

Race, $\uparrow n(\%)$

$\begin{array}{lllll}\text { Black } & 0 & 1(10) & 1(10) & 2(6.7)\end{array}$

$\begin{array}{lllll}\text { White } & 9(90) & 9(90) & 9(90) & 27(90)\end{array}$

$\begin{array}{llllll}\text { Hispanic } & 1(10) & 0 & 0 & 1(3.3) & 0\end{array}$

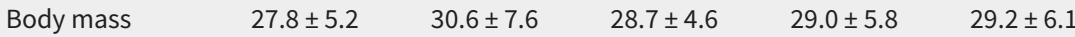

Note: pfu = plaque-forming units, $S D=$ standard deviation.

*Plus-minus values are means \pm SD. There were no significant differences between groups. 
Real-time reverse-transcriptase polymerase chain reaction (RT-PCR) was performed to identify vaccine rVSV in plasma, saliva or urine through amplification of the VSV-nucleoprotein gene. ${ }^{16}$

Routine hematologic, biochemistry and screening serology were performed at the IWK Health Centre using standard methods.

\section{Statistical analysis}

Categorical variables were summarized by number and percentage of participants within each category (with a category for missing data) of the parameter. For continuous variables, the number of participants, mean (or geometric mean, where applicable), median, standard deviation (SD) and 95\% confidence interval (CI) for the geometric mean, minimum and maximum values were performed. Statistical hypothesis testing of primary and secondary immunogenicity outcomes was conducted at a 2-sided 0.05 significance level; $p$ values were not adjusted for multiplicity. Summary statistics were performed as well as 2-sided $95 \% \mathrm{Cls}$ on selected parameters. An analysis of variance (ANOVA) regression model was performed to

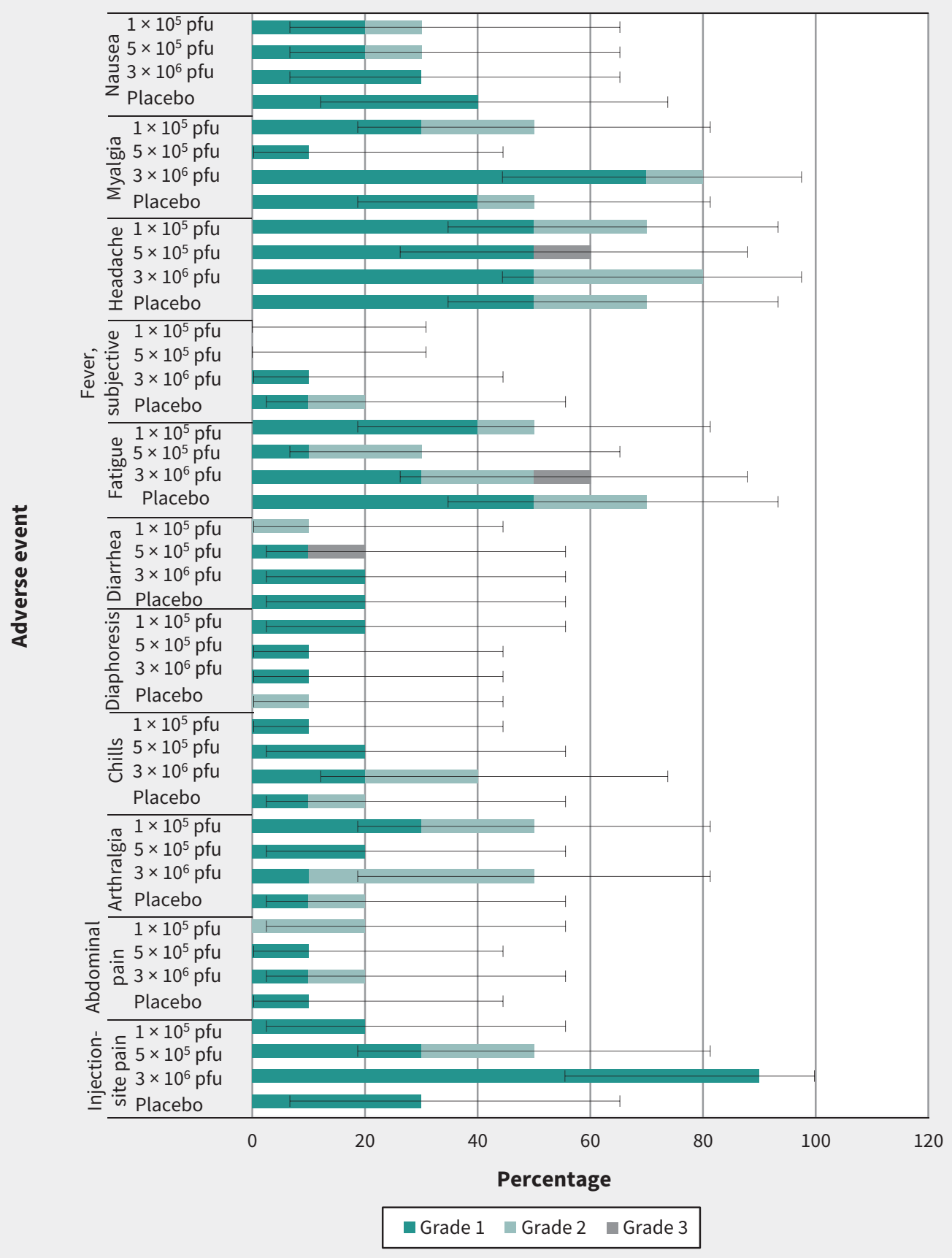

Figure 2: Frequency of local and systemic adverse events (AEs) among vaccine cohorts or placebo. Note: Solicited AEs and their severity reported in the 14 days postinjection for vaccine doses of $1 \times 10^{5}$ plaque-forming units (pfu) (cohort 1 ), $5 \times 10^{5}$ pfu (cohort 2), $3 \times 10^{6}$ pfu (cohort 3 ) and placebo shown with $95 \%$ confidence intervals for any grade of AEs within each group. 
compare log (base 10) transformed ZEBOV IgG concentrations (in ELISA units/mL); pairwise comparisons of antibody concentrations at analysis days 14, 28 and 180 between rVSV $\Delta$ G-ZEBOV-GP dose levels and between each rVSV $\triangle$ G-ZEBOV-GP dose level with placebo were performed in the per-protocol population. The ELISA lower limit of quantification was 55.34 units/mL; samples with a concentration at or below the lower limit of quantification were entered as 27.67 for statistical analysis. PsVNA positivity was defined by titers of 20 or higher; values $<20$ were assigned 10 for calculation. For both assays, seroconversion was defined as at least 4-fold increase from baseline titer. Antibody titer calculations were on the $\log _{10}$ scale. Summaries were provided for each vaccine dose and for placebo.

All descriptive statistical analyses were generated using SAS software version 9.3 or later. (SAS and all other SAS Institute Inc. product or service names are registered trademarks or trademarks of SAS Institute Inc., Cary, NC.) Medical history and AEs were coded using the Medical Dictionary for Regulatory Activities (MedDRA) version 17.0. Concomitant medications were coded using the most recently available version of the World Health Organization Drug Dictionary.

\section{Ethics approval}

Written, informed consent was obtained before any study procedure. The protocol was approved by Health Canada and the Research Ethics Boards of the IWK Health Centre and the Public Health Agency of Canada.

\section{Results}

\section{Study participants}

A total of 40 participants were vaccinated between Nov. 27 and Dec. 15, 2014 (Table 1). Mean age was 35.6 years (range 18-62) among vaccine recipients, and 32.0 years (range 20-50) among placebo recipients; 23 were female $(57.5 \%)$. All participants completed follow-up visits (Figure 1).

\section{Safety}

There were no SAEs related to the vaccine or withdrawals from the study. The $3 \mathrm{SAE}$ reports from 2 study subjects were cholelithiasis in a recipient of $5 \times 10^{5}$ pfu vaccine, and psychotic disorder and major depression in a placebo recipient. All were assessed as being unrelated to the vaccine, and were resolved. Reports of AEs solicited from the participants were primarily characterized as mild to moderate, with 3 separate severe events: headache and diarrhea in the $5 \times 10^{5}$ group, and fatigue in the $3 \times 10^{6}$ group. Headache was the most frequent systemic reaction overall (Figure 2). There were no reports of injection-site swelling or redness; only mild to moderate pain was noted locally. In the first day postvaccination, there was higher incidence of injection-site pain in vaccine recipients compared with placebo. Incidence of injectionsite pain was $0 \%, 30 \%$ and $80 \%$ in the $1 \times 10^{5} \mathrm{pfu}, 5 \times 10^{5} \mathrm{pfu}$ and $3 \times$ $10^{6}$ pfu cohorts, respectively, and $20 \%$ in placebo.

Arthralgia and myalgia had a similar trend, with frequency on the first day postvaccination higher in vaccine than in placebo recipients. Arthralgia was reported in $10 \%, 0 \%$ and $50 \%$ in the $1 \times$ $10^{5} \mathrm{pfu}, 5 \times 10^{5} \mathrm{pfu}$, and $3 \times 10^{6} \mathrm{pfu}$ dose cohorts, respectively, and 0 in placebo; and the incidence of myalgia was $20 \%, 0 \%$ and $40 \%$ in the $1 \times 10^{5} \mathrm{pfu}, 5 \times 10^{5} \mathrm{pfu}$ and $3 \times 10^{6} \mathrm{pfu}$ cohorts, respectively, and $10 \%$ in placebo. Onset of systemic solicited AEs ranged from as early as the day of injection to 14 days thereafter, lasting 1 to 9 days. Objective fever was not measured in any of the participants. A grade 1 subjective fever (3.3\%) was reported by 1 participant who received the $3 \times 10^{6}$ pfu dose, and 2 placebo recipients reported grade 1 and grade 2 subjective fever.

Overall, $60 \%$ of vaccinees reported unsolicited AEs, yet the incidence of each event was not greater than 2 subjects in any treatment arm (data not shown). Unsolicited AEs were mostly mild to moderate, except a report of severe arthralgia that started at day 18 and lasted 1 day, with no objective signs of arthritis upon immediate examination. No cases of arthritis were observed; however, 1 subject receiving the $3 \times 10^{6}$ pfu dose reported grade 1 joint swelling for 14 days, starting at day 13 postvaccination.

\section{Detection of viremia and virus shedding by PCR}

Two participants in the $3 \times 10^{6} \mathrm{pfu}$ group developed viremia by the first day postvaccination, which continued to day 3 for 1 of them. Overall, viremia peaked on study day 3, when 18 (60\%) of the 30 vaccinated participants were positive, with the greatest frequency in the highest dose group. All subsequent samples were negative (Table 2). There was no virus shedding in saliva or urine.

Table 2: Polymerase chain reaction detection of rVSVAG-ZEBOV-GP vaccine virus

\begin{tabular}{|c|c|c|c|c|c|}
\hline \multirow{2}{*}{$\begin{array}{l}\text { Study } \\
\text { day }\end{array}$} & \multirow{2}{*}{$\begin{array}{l}\text { Specimen } \\
\text { type }\end{array}$} & $\begin{array}{c}\text { Vaccine, } \\
1 \times 10^{5} \mathrm{pfu} \\
(n=10)\end{array}$ & $\begin{array}{l}\text { Vaccine, } \\
5 \times 10^{5} \mathrm{pfu} \\
(n=10)\end{array}$ & $\begin{array}{l}\text { Vaccine, } \\
3 \times 10^{6} \mathrm{pfu} \\
(n=10)\end{array}$ & $\begin{array}{l}\text { All vaccine } \\
\text { subjects } \\
(n=30)\end{array}$ \\
\hline & & \multicolumn{4}{|c|}{ No. of positive samples/no. of samples tested (\%) } \\
\hline \multirow[t]{3}{*}{1} & Blood & 0 & 0 & $2 / 10(20)$ & $2 / 30(6.7)$ \\
\hline & Saliva & 0 & 0 & 0 & 0 \\
\hline & Urine & 0 & 0 & 0 & 0 \\
\hline \multirow[t]{3}{*}{3} & Blood & $5 / 10(50)$ & $5 / 10(50)$ & $8 / 10(80)$ & $18 / 30(60)$ \\
\hline & Saliva & 0 & 0 & 0 & 0 \\
\hline & Urine & 0 & 0 & 0 & 0 \\
\hline \multirow[t]{3}{*}{7} & Blood & 0 & 0 & 0 & 0 \\
\hline & Saliva & 0 & 0 & 0 & 0 \\
\hline & Urine & 0 & 0 & 0 & 0 \\
\hline \multirow[t]{3}{*}{14} & Blood & 0 & 0 & 0 & 0 \\
\hline & Saliva & 0 & 0 & 0 & 0 \\
\hline & Urine & 0 & 0 & 0 & 0 \\
\hline
\end{tabular}

Note: Study day is relative to vaccination day, which is study day 0 . pfu = plaque-forming units. 
Immunogenicity

\section{ZEBOV rGP ELISA}

Seroconversions were observed by day 14 in all 3 vaccine dose groups, 4 of $10(40 \%)$ who received the $1 \times 10^{5} \mathrm{pfu}, 2$ of $10(20 \%)$ who received the $5 \times 10^{5} \mathrm{pfu}$, and 4 of $10(40 \%)$ who received the $3 \times 10^{6}$ pfu doses (Table 3). By day 28 , seroconversions had increased to 7 of $10(70 \%)$ who received the $1 \times 10^{5}$ pfu or $5 \times 10^{5}$ $\mathrm{pfu}$, and all $10(100 \%)$ who received the $3 \times 10^{6} \mathrm{pfu}$ doses. With the exception of 1 subject in the $1 \times 10^{5}$ pfu group, all vaccinees showed seroconversion at some point during the course of the study. Geometric mean titers (GMTs) increased over time from day 0 to day 180 , with the exception of a decrease in the $3 \times 10^{6}$ pfu dose group from day 28 to day 180 . At day 28 , there was a nonsignificant trend to higher GMTs in the $3 \times 10^{6} \mathrm{pfu}$ group compared with the low and medium doses $(p=0.071$ and $p=$ 0.054 , respectively). Serum IgG titers at study day 180 remained significantly higher in all 3 dose groups compared with placebo. GMT responses were similar in the $1 \times 10^{5} \mathrm{pfu}$ and $5 \times 10^{5} \mathrm{pfu}$ dose groups.

\section{Table 3: Geometric mean antibody titers to Ebola glycoprotein}

\begin{tabular}{|c|c|c|c|c|c|c|c|c|}
\hline \multirow{2}{*}{$\begin{array}{l}\text { Dose/ } \\
\text { study } \\
\text { day }\end{array}$} & \multirow{2}{*}{$\begin{array}{c}\text { No. of } \\
\text { participants }\end{array}$} & \multirow[b]{2}{*}{ GMT $(95 \% \mathrm{CI})$} & \multicolumn{4}{|c|}{$p$ value for GMT/day 28} & \multicolumn{2}{|c|}{ Seroconversion } \\
\hline & & & Placebo & $1 \times 10^{5} \mathrm{pfu}$ & $5 \times 10^{5} \mathrm{pfu}$ & $3 \times 10^{6} \mathrm{pfu}$ & $n(\%)$ & $p$ value \\
\hline \multicolumn{9}{|c|}{$1 \times 10^{5} \mathrm{pfu}$} \\
\hline 0 & 10 & $27.7(-)$ & & & & & NA & NA \\
\hline 7 & 10 & $27.7(-)$ & & & & & 0 & 1.0 \\
\hline 14 & 10 & $96.8(36.9-253.7)$ & 0.015 & NA & 0.790 & 0.188 & $4(40)$ & 0.087 \\
\hline 28 & 10 & $636.2(258.6-1565.2)$ & $<0.001$ & NA & 0.894 & 0.071 & $7(70)$ & 0.003 \\
\hline 56 & 10 & 824.9 (341.7-1991.4) & & & & & $8(80)$ & $<0.001$ \\
\hline 84 & 10 & $993.6(469.6-2102.1)$ & & & & & $9(90)$ & $<0.001$ \\
\hline 180 & 9 & 1169.7 (586.4-2333.2) & $<0.001$ & NA & 0.537 & 0.488 & $8(88.9)$ & $<0.001$ \\
\hline \multicolumn{9}{|c|}{$5 \times 10^{5} \mathrm{pfu}$} \\
\hline 0 & 10 & $27.7(-)$ & & & & & NA & NA \\
\hline 7 & 10 & $27.7(-)$ & & & & & 0 & 1.0 \\
\hline 14 & 10 & $110.5(34.0-359.1)$ & 0.008 & 0.790 & NA & 0.290 & $2(20)$ & 0.474 \\
\hline 28 & 10 & $603.5(286.0-1273.5)$ & $<0.001$ & 0.894 & NA & 0.054 & $7(70)$ & 0.003 \\
\hline 56 & 10 & $792.8(279.1-2252.2)$ & & & & & $8(80)$ & $<0.001$ \\
\hline 84 & 10 & $876.7(410.1-1874.3)$ & & & & & $8(80)$ & $<0.001$ \\
\hline 180 & 10 & $928.1(481.4-1789.4)$ & $<0.001$ & 0.537 & NA & 0.924 & $10(100)$ & $<0.001$ \\
\hline \multicolumn{9}{|c|}{$3 \times 10^{6} \mathrm{pfu}$} \\
\hline 0 & 10 & $27.7(-)$ & & & & & NA & NA \\
\hline 7 & 10 & $27.7(-)$ & & & & & 0 & 1.0 \\
\hline 14 & 10 & $187.3(126.0-278.4)$ & $<0.001$ & 0.188 & 0.290 & NA & $4(40)$ & 0.087 \\
\hline 28 & 10 & $1321.3(830.2-2102.9)$ & $<0.001$ & 0.071 & 0.054 & NA & $10(100)$ & $<0.001$ \\
\hline 56 & 10 & $1152.6(771.3-1722.5)$ & & & & & $10(100)$ & $<0.001$ \\
\hline 84 & 9 & $992.6(583.3-1689.1)$ & & & & & $9(100)$ & $<0.001$ \\
\hline 180 & 9 & $895.7(437.2-1835.1)$ & $<0.001$ & 0.488 & 0.924 & NA & $8(88.9)$ & $<0.001$ \\
\hline \multicolumn{9}{|c|}{ Placebo } \\
\hline 0 & 10 & $27.7(-)$ & & & & & NA & NA \\
\hline 7 & 10 & $27.7(-)$ & & & & & NA & NA \\
\hline 14 & 10 & $27.7(-)$ & NA & 0.015 & 0.008 & $<0.001$ & NA & NA \\
\hline 28 & 10 & $27.7(-)$ & NA & $<0.001$ & $<0.001$ & $<0.001$ & NA & NA \\
\hline 56 & 9 & $27.7(-)$ & & & & & NA & NA \\
\hline 84 & 10 & $27.7(-)$ & & & & & NA & NA \\
\hline 180 & 10 & $30.9(24.0-39.8)$ & NA & $<0.001$ & $<0.001$ & $<0.001$ & NA & NA \\
\hline
\end{tabular}

Note: $\mathrm{Cl}=$ confidence interval, $\mathrm{GMT}=$ geometric mean titers, $\mathrm{NA}=$ not applicable, pfu = plaque-forming units 


\section{PsVNA titers}

Seroconversion by day 28 using PsVNA50 was noted in 8 of 10 $(80 \%)$ volunteers in the $1 \times 10^{5}$ and $5 \times 10^{5}$ pfu groups, and in 7 of $10(70 \%)$ of the volunteers in the $3 \times 10^{6}$ group (Figure 3$)$. On day 28 , seroconversion by PsVNA80 was less, with rates of $20 \%$ (2 of 10 volunteers) in the $1 \times 10^{5}$ and $5 \times 10^{5} \mathrm{pfu}$ groups, and in 1 of 10 $(10 \%)$ of the volunteers in the $3 \times 10^{6}$ group. No difference was observed at baseline between vaccine groups and placebo.

\section{Interpretation}

In this phase 1 study, all 3 dose levels of rVSV $\Delta$ G-ZEBOV-GP live, attenuated vaccine were well tolerated by participants and no safety concerns were identified. Solicited AEs were primarily characterized as mild to moderate, with only 3 severe events (headache and diarrhea in the $5 \times 10^{5}$ pfu group; fatigue in the 3 $\times 10^{6}$ pfu group). Arthralgia during the first 14 days postvaccination was infrequent and not severe. Arthritis was not reported. Viremia was transient, with no detection of vaccine virus shedding in urine or saliva. In the subset of vaccinees who developed viremia, the incidence of frequently reported solicited AEs was similar or moderately increased compared with other vaccinated subjects overall. The vaccine was immunogenic, eliciting glycoprotein-binding antibodies in recipients of all 3 doses. In this report, we provide the first extended postvaccination serology assessments and show that antibody titers persist at high levels 180 days postimmunization.

A phase 1 , dose-escalation trial at WRAIR enrolled participants to assess the safety and immunogenicity of $3 \times 10^{6} \mathrm{pfu}$ and $2 \times 10^{7}$ pfu doses. ${ }^{16}$ At the United States National Institutes of Health (NIH) Clinical Center, a 2-dose regimen was evaluated using these 2 dose levels. ${ }^{16}$ The VSV Ebola Consortium ${ }^{25}$ conducted 4 parallel phase 1 trials at sites within Europe and Africa. ${ }^{24}$ Three of the 4 trials were open label, uncontrolled and dose escalating, designed to assess the safety, AEs and immunogenicity of rVSV $\Delta \mathrm{G}$ ZEBOV-GP doses ranging from $3 \times 10^{5}$ to $2 \times 10^{7} \mathrm{pfu}$. The fourth trial, in Geneva, initially administered the $1 \times 10^{7} \mathrm{pfu}$ and $5 \times 10^{7}$ pfu vaccine doses, but was put on hold as a result of a $25 \%$ (13 of 51 ) and $22 \%$ (11 of 51) incidence of fever and oligoarthritis with these 2 doses, respectively. After preliminary data indicated tolerability and immunogenicity of low vaccine doses in other studies, the study was resumed in Geneva, but at a lower dose of $3 \times 10^{5}$ pfu, comparing outcomes to those of vaccinees who had received the higher doses before the study was put on hold. ${ }^{26}$

Patterns of detectable viremia were comparable with other studies that investigated the rVSV $\Delta$ G-ZEBOV-GP vaccine; viremia was transient, peaking by day 3 and no longer evident on day 7. 16,24,26 Glycoprotein-binding serum antibodies were detectable in all vaccinated individuals, irrespective of vaccine dose. Although neutralizing antibody titers were detected among most participants of our study, a dose response was not evident. A significant dose response for neutralizing antibody titers was not observed in the WRAIR phase 1 trial comparing $3 \times 10^{6}$ versus $2 \times$ $10^{7} \mathrm{pfu}$ dose, but was observed in the larger VSV Ebola Consortium studies comparing multiple doses between $3 \times 10^{5}$ and $5 \times$ $10^{7}$ pfu dose with higher vaccine doses. ${ }^{16,24,26}$ Unlike higher doses of the rVSV $\Delta$ G-ZEBOV-GP vaccine, ${ }^{24}$ the dose levels tested in our study were minimally reactogenic, which confirms the tolerability of low-dose rVSV $\Delta$ G-ZEBOV-GP vaccination. ${ }^{16,24,26}$ There were no reports of arthritis among our cohort for the 180-day followup period, similar to published trials from WRAIR and NIH. ${ }^{16}$ Reports of arthritis with overlapping and higher doses of the vaccine suggest an intrinsic association with rVSV $\triangle$ G-ZEBOV-GP, and not only a dose-dependent one..$^{24,26}$

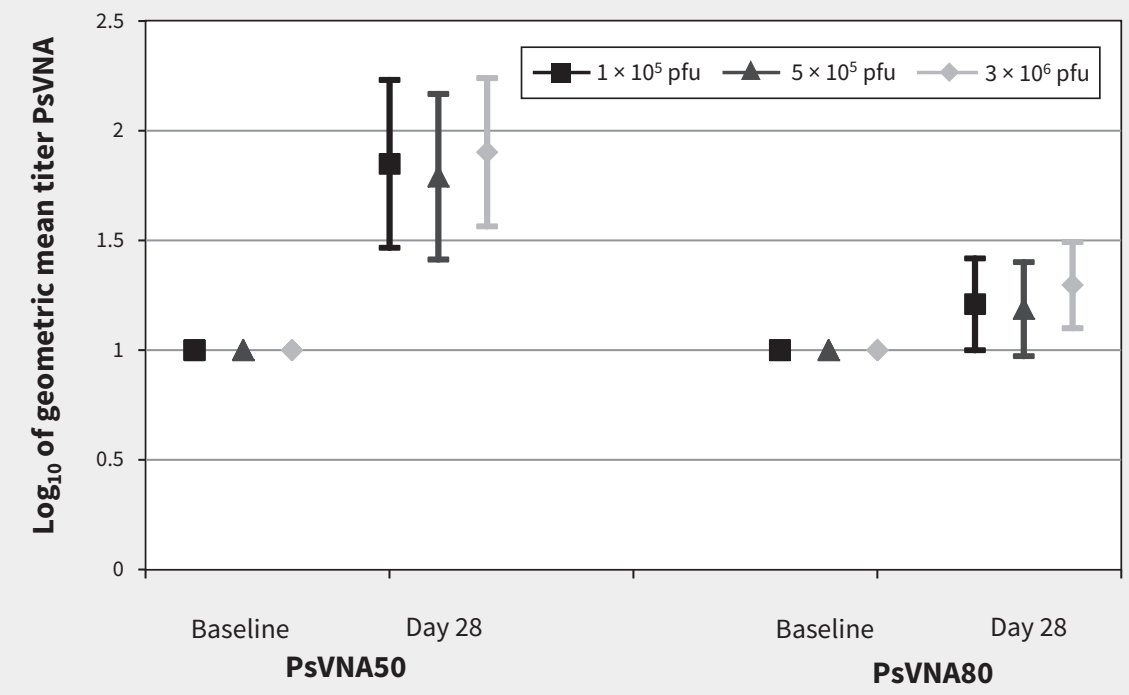

Figure 3: Neutralization antibody responses to Ebola glycoprotein. Note: There were no significant differences between groups using the pseudovirion neutralization assay (PsVNA) at day 28. The PsVNA50 of the $3 \times$ $10^{6}$ plaque-forming units ( $\mathrm{pfu}$ ) dose compared with the $1 \times 10^{5} \mathrm{pfu}$ and $5 \times 10^{5}$ pfu doses was $p=0.788$ and $p=$ 0.575 , respectively. 
The multiple phase 1 rVSV $\Delta$ G-ZEBOV-GP trials across North America (United States and Canada), Europe (Switzerland and Germany) and nonepidemic regions of Africa (Gabon and Kenya) have shown that the vaccine is well tolerated and immunogenic, and warrants further study. The range of doses administered in this study were assessed along with those in the WRAIR and NIH studies and together used to select the optimum dose to be evaluated in the phase $2 / 3$ trials. Currently, there are 4 ongoing phase 2/3 studies: a phase 2 placebo-controlled trial in Liberia, sponsored by the US NIH; a phase 3 ring vaccination trial in Guinea, sponsored by the World Health Organization; a phase 3 trial in Sierra Leone, sponsored by the US Centers for Disease Control and Prevention; and a phase 3 immunogenicity, safety and lot consistency trial in North America and Europe, sponsored by Merck. As part of an African-Canadian collaboration, preparations for a phase 2 trial evaluating the safety and immunogenicity of rVSV $\Delta$ G-ZEBOV-GP in HIV-infected adults and adolescents are underway with the intent to begin enrolling in 2017.

There are several candidate Ebola vaccines at different stages of development and testing. ${ }^{27}$ The rVSV $\Delta$ G-ZEBOV-GP vaccine is the first to progress to phase 3 trials in Africa after data from the 8 phase 1 studies, including this one, collectively determined that $2 \times 10^{7} \mathrm{pfu}$ is a favourable dose. Other vaccines include the Ad26-EBOV and MVA-EBOV by Johnson \& Johnson and Bavarian Nordic, which have entered phase 2 and 3 trials. The recombinant protein Ebola vaccine by Novavax and Monovalent Ebola Zaire Vaccine (rVSVN4CT1-EBOVGP1) by Profectus BioSciences Inc. are being assessed in phase 1 trials. The replication-defective chimpanzee adenovirus 3 ( $\mathrm{ChAd} 3$ ) is completing phase 2 studies. ${ }^{28-30}$

\section{Limitations}

Limitations of this study are primarily the small sample size, overall and per group, and the fact that only participants from North America were enrolled. Another limitation is the lack of follow-up after six months for long-term immunogenicity data.

\section{Conclusion}

Ebola transmission in Africa is now considered to be under control. ${ }^{2,31}$ However, recent clusters of cases are still being reported as a result of virus spread from shedding survivors, and more are anticipated, with the virus still present in sub-Saharan Africa's ecosystem. ${ }^{32}$ These facts underscore the importance of continuing efforts and collaborations that may ultimately lead to licensed Ebola vaccines that would protect humans and prevent or control outbreaks in the future. Our study confirms the immunogenicity and tolerability of the rVSV $\triangle$ G-ZEBOV-GP vaccine, and provides new information about duration of the antibody response.

\section{References}

1. Urbanowicz RA, McClure CP, Sakuntabhai A, et al. Human adaptation of Ebola virus during the West African outbreak. Cell 2016;167:1079-87.e5.

2. Ebola virus disease [situation report]. Geneva: World Health Organization; 2016. Available: http://apps.who.int/iris/bitstream/10665/208883/1/ebolasitrep _10Jun2016_eng.pdf?ua=1 (accessed 2017 Jan. 20).
3. Garbutt M, Liebscher R, Wahl-Jensen V, et al. Properties of replication-competent vesicular stomatitis virus vectors expressing glycoproteins of filoviruses and arenaviruses. J Virol 2004;78:5458-65.

4. Schnell MJ, Buonocore L, Kretzschmar E, et al. Foreign glycoproteins expressed from recombinant vesicular stomatitis viruses are incorporated efficiently into virus particles. Proc Natl Acad Sci U S A 1996;93:11359-65.

5. Letchworth GJ, Rodriguez LL. Del cbarrera J. Vesicular stomatitis. Vet J 1999; 157:239-60.

6. Lichty BD, Power AT, Stojdl DF, et al. Vesicular stomatitis virus: re-inventing the bullet. Trends Mol Med 2004;10:210-6.

7. Takada A, Feldmann H, Stroeher U, et al. Identification of protective epitopes on ebola virus glycoprotein at the single amino acid level by using recombinant vesicular stomatitis viruses. J Virol 2003;77:1069-74.

8. Mire CE, Miller AD, Carville A, et al. Recombinant vesicular stomatitis virus vaccine vectors expressing filovirus glycoproteins lack neurovirulence in nonhuman primates. PLoS Negl Trop Dis 2012;6:e1567.

9. Watanabe S, Takada A, Watanabe T, et al. Functional importance of the coiled-coil of the Ebola virus glycoprotein. J Virol 2000;74:10194-201.

10. Feldmann H, Jones SM, Daddario-DiCaprio KM, et al. Effective post-exposure treatment of Ebola infection. PLOS Pathog 2007;3:e2.

11. Daddario-DiCaprio KM, Geisbert TW, Stroher U, et al. Postexposure protection against Marburg haemorrhagic fever with recombinant vesicular stomatitis virus vectors in non-human primates: an efficacy assessment. Lancet 2006; 367:1399-404.

12. Geisbert TW, Daddario-Dicaprio KM, Lewis MG, et al. Vesicular stomatitis virusbased ebola vaccine is well-tolerated and protects immunocompromised nonhuman primates. PLoS Pathog 2008;4:e1000225.

13. Geisbert TW, Daddario-Dicaprio KM, Geisbert JB, et al. Vesicular stomatitis virusbased vaccines protect nonhuman primates against aerosol challenge with Ebola and Marburg viruses. Vaccine 2008;26:6894-900.

14. Jones SM, Feldmann $\mathrm{H}$, Ströher $\mathrm{U}$, et al. Live attenuated recombinant vaccine protects nonhuman primates against Ebola and Marburg viruses. Nat Med 2005;11:786-90.

15. Guideline for good clinical practice E6(R2): step 5. London (UK): European Medicines Agency; 2016. Available: www.ema.europa.eu/docs/en_GB/document_ library/Scientific_guideline/2009/09/WC500002874.pdf (accessed 2017 Mar. 9).

16. Regules JA, Beigel JH, Paolino KM, et al. rVSVAG-ZEBOV-GP Study Group. A recombinant vesicular stomatitis virus Ebola vaccine. N Engl J Med 2017;376:330-41.

17. Rudge TL Jr, Badorrek C, Rodriguez N, et al. Qualification and validation of filovirus anti-glycoprotein immunoglobulin ELISAs. Abstract at Chemical and Biological Defense Program 2015, DTRA: rVSV Ebola Vaccine Science Review; July 2015; Arlington, Va.

18. Rudge TL Jr, Sankovich K, Kurnat K, et al. Development and qualification of a human filovirus anti-glycoprotein immunoglobulin ELISAs. Abstract at the 7th International Symposium on Filoviruses; March 2015; Washington, DC.

19. Sabourin CL, Rudge TL Jr, Gainey M, et al. Development and qualification of filovirus anti-glycoprotein immunoglobulin ELISAs. Abstract at the 6th International Symposium on Filoviruses; March-April 2014; Galveston, Tex.

20. Ray N, Whidby J, Stewart S, et al. Study of Andes virus entry and neutralization using a pseudovirion system. J Virol Methods 2010;163:416-23.

21. Ogino $\mathrm{M}$, Ebihara $\mathrm{H}$, Lee $\mathrm{BH}$, et al. Use of vesicular stomatitis virus pseudotypes bearing hantaan or seoul virus envelope proteins in a rapid and safe neutralization test. Clin Diagn Lab Immunol 2003;10:154-60.

22. Grant-Klein RJ, Van Deusen NM, Badger CV, et al. A multiagent filovirus DNA vaccine delivered by intramuscular electroporation completely protects mice from ebola and Marburg virus challenge. Hum Vaccin Immunother 2012;8:1703-6.

23. Kwilas S, Kishimori JM, Josleyn M, et al. A hantavirus pulmonary syndrome (HPS) DNA vaccine delivered using a spring-powered jet injector elicits a potent neutralizing antibody response in rabbits and nonhuman primates. Curr Gene Ther 2014;14:200-10.

24. Agnandji ST, Huttner A, Zinser ME, et al. Phase 1 trials of rVSV Ebola vaccine in Africa and Europe. N Engl J Med 2016;374:1647-60. 
25. Kanapathipillai R, Henao Restrepo AM, Fast $P$, et al. Ebola vaccine - an urgent international priority. N Engl J Med 2014;371:2249-51.

26. Huttner A, Dayer JA, Yerly S, et al. VSV-Ebola Consortium. The effect of dose on the safety and immunogenicity of the VSV Ebola candidate vaccine: a randomised doubleblind, placebo-controlled phase 1/2 trial. Lancet Infect Dis 2015;15:1156-66.

27. Pavot V. Leading Ebola vaccine candidates. Vaccin Res Open J 2016;1:1-6.

28. GlaxoSmithKline. Safety and immunogenicity study of GSK biologicals' investigational recombinant chimpanzee adenovirus type 3-vectored Ebola Zaire vaccine (GSK3390107A) in children in Africa. In: ClinicalTrials.gov [Internet]. Bethesda (MD): National Library of Medicine (US); 2000. Available: https://clinicaltrials.gov/ct2/ show/NCT02548078 (accessed 2016 Nov. 3). NLM identifier: NCT02548078.

29. GlaxoSmithKline. Safety and immunogenicity study of GSK biologicals' investigational recombinant chimpanzee adenovirus type 3-vectored Ebola Zaire vac- cine (GSK3390107A) in adults in Africa. In: ClinicalTrials.gov [Internet]. Bethesda (MD): National Library of Medicine (US); 2000. Available: https://clinicaltrials.gov/ ct2/show/NCT02485301 (accessed 2016 Nov. 3). NLM identifier: NCT02485301.

30. National Institute of Allergy and Infectious Diseases. Partnership for Research on Ebola Vaccines in Liberia (PREVAIL). In: ClinicalTrials.gov [Internet]. Bethesda (MD): National Library of Medicine (US); 2000. Available: https://clinicaltrials.gov/ ct2/show/NCT02344407 (accessed 2016 Nov. 3). NLM identifier: NCT02344407.

31. Shiwani HA, Pharithi RB, Khan B, et al. An update on the 2014 Ebola outbreak in Western Africa. Asian Pac J Trop Med 2017;10:6-10.

32. Statement on the 9th meeting of the IHR Emergency Committee regarding the Ebola outbreak in West Africa [statement]. Geneva: World Health Organization; 2016. Available: www.who.int/mediacentre/news/statements/2016/end-ofebola-pheic/ en/ (accessed 2017 Mar. 10).
Competing interests: May ElSherif reports grants from the Canadian Institutes of Health Research/Public Health Agency of Canada, during the conduct of the study. Donna Mackinnon-Cameron reports an operating grant from the Canadian Institutes of Health Research/Public Health Agency of Canada, during the conduct of the study. Li Li reports an operating grant from the Canadian Institutes of Health Research/Public Health Agency of Canada, during the conduct of the study. Judie Alimonti reports personal fees from NewLink Genetics, outside the submitted work; and Her Majesty the Queen in right of Canada holds a patent related to the rVSVIG-ZEBOV-GP vaccine (patents: EU 1527087; US 8012489; CA $2,493,142)$. W. Jay Ramsey was an employee of NewLink Genetics during the conduct of the study, and reports personal fees as a salaried employee of NewLink Genetics, outside the submitted work. Donald Gray Heppner reports grants from the Department of Health and Human Services, during the conduct of the study and employment by NewLink Genetics Inc. (which is developing the vaccine described in this article), outside the submitted work. Tracy Kemp was an employee of NewLink Genetics during the conduct of the study; she also reports personal fees as a salaried employee of NewLink Genetics, outside the submitted work. Thomas Monath reports a grant from BARDA and the US Department of Health and Human Services, and financial support for study, personal salary and benefits from NewLink Genetics. Shelly McNeil reports an operating grant from the Canadian Institutes of Health Research/Public Health Agency of Canada, during the conduct of the study; and grants and personal fees from vaccine manufacturers (such as Merck, GlaxoSmithKline Biologicals, Sanofi Pasteur and Pfizer) outside the submitted work. Joanne Langley reports an operating grant from the Canadian Institutes of Health Research/Public Health Agency of Canada, during the conduct of the study, as well as grants and personal fees from vaccine manufacturers (such as Merck, GlaxoSmithKline Biologicals, Sanofi Pasteur, Pfizer, and Novartis) to conduct vaccine clinical trials, outside the submitted work. Scott Halperin reports an operating grant from the Canadian Institutes of Health Research/Public Health Agency of Canada, during the conduct of the study, and grants and personal fees from vaccine manufacturers (such as Merck, GlaxoSmithKline Biologicals, Sanofi Pasteur, Pfizer and Novartis) outside the submitted work. No other competing interests were declared.

This article has been peer reviewed.

Affiliations: Canadian Center for Vaccinology (EISherif, Brown, MacKinnon-Cameron, Li, McNeil, Langley, Halperin), IWK Health Centre and Nova Scotia Health Authority, Dalhousie University, Halifax, NS; National Microbiology Laboratory (Racine, Alimonti), Winnipeg, Man.; Battelle Biomedical Research Center (Rudge, Sabourin), Columbus, Ohio; United States Army Medical Research Institute of Infectious Disease (Silvera, Hooper, Kwilas), Fort Detrick, Md.; Joint Program Executive Office for Chemical and Biological Defense Medical Countermeasure Systems' Joint Vaccine Acquisition Program (Kilgore, Badorrek), Fort Detrick, Md.; BioProtection Systems/ NewLink Genetics Corporation (Ramsey, Heppner, Kemp, Monath), Ames, lowa; Veristat LLC (Nowak), Southborough, Mass.

Contributors: All authors approved the final version of this manuscript for publication and agreed to be accountable. All authors either drafted and/or revised the work. All authors contributed to one or more of the following: i) conception/design of the work; ii) data acquisition; iii) data analysis; iv) data interpretation.

Funding: Funding was provided by the Public Health Agency of Canada and the Canadian Institutes of Health Research through a grant to the Canadian Immunization Research Network (CIRN), Funding Reference Number
FRN\#138434, and administered by Dalhousie University. Funding was also provided by the US Department of Defense Joint Program Executive Office for Chemical and Biological Defense Medical Countermeasure Systems' Joint Vaccine Acquisition Program (MCS-JVAP) under contracts to the US Army Medical Research Institute of Infectious Disease (USAMRIID) and Battelle Biomedical Research Center. This research was supported in part by an appointment to the Postgraduate Research Participation Program at the USAMRIID, administered by the Oak Ridge Institute for Science and Education through an interagency agreement between the US Department of Energy and US Army Medical Research and Materiel Command.

Data sharing: Data from this study are the property of the Dalhousie University on behalf of the Canadian Immunization Research Network through agreements with funding partners. Data are available to others, through direct request to Scott Halperin at the Canadian Center for Vaccinology, IWK Health Centre, Halifax, NS; email: scott.halperin@dal.ca. Access data will be provided by sharing tables, figures or summary listings as needed by interested parties.

Acknowledgements: The authors would like to thank Canadian Center for Vaccinology staff; Marilyn Tiller, IWK Research Pharmacist; and Brian Martin, Julianne Creager, Rick Nichols, Joan Fusco, Charles Link and Nick Vahanian from NewLink Genetics.

Disclaimer: The opinions, interpretations, conclusions and recommendations contained herein are those of the authors and are not necessarily endorsed by the US Department of Defense.

Accepted: May 8, 2017

Correspondence to: Scott Halperin, scott.halperin@dal.ca 